\title{
Нейросетевое моделирование содержания хлороформа в питьевой воде при водоподготовке
}

\author{
А. И. Юхно, Н. К. Плуготаренко, К.Т.н., ФГАОУ ВО «Южный федеральный университет» \\ a.bachmackaja@gmail.com
}

УДК 51.7

Работа посвящена применению нейросетевого моделирования в сфере водоподготовки. Разработана нейросетевая модель, способная прогнозировать концентрации хлороформа, образующиеся в ходе хлорирования питьевой воды, в зависимости от характеристик качества воды. Проведена серия компьютерных экспериментов по исследованию влияния отдельных факторов на целевой параметр. Построенная нейросетевая модель дает возможность создания системы контроля над содержанием хлорорганических соединений в питьевой воде, тем самым обеспечивая условия более безопасного водопотребления.

Большинству функционирующих станций обеззараживания питьевой воды, спроектированных в соответствии с традиционными технологиями осветления и хлорирования воды, становится все труднее справляться с постоянно растущим антропогенным загрязнением водоисточников [1-2].

В таких условиях применение нейросетевого моделирования для выявления аномалий качества питьевой воды, а также для подбора оптимальных параметров и условий подготовки питьевой воды является чрезвычайно актуальным [3-7]. Моделирование свойств воды и параметров водоподготовки позволяет осуществить целенаправленный поиск оптимальных характеристик технологического процесса хлорирования питьевой воды, при которых образуется наименьшее количество канцерогенных соединений и тем самым обеспечить условия более безопасного водопотребления.

\section{ОПИСАНИЕ МОДЕЛИ}

Для установления зависимости между значениями параметров, определяющих качество питьевой воды, и концентрациями хлорорганических соединений использовали метод наименьших квадратов (МНК), для расчета применяли пакет программ Maple 13. Из числа исследуемых параметров удалось исключить цветность и мутность, так как эти характеристики качества не оказывают значительного влияния на образование галогенорганических соединений в процессе хлорирования питьевой воды.

Для построения нейронной сети (НС), моделирующей влияние параметров процесса обеззараживания воды и ее исходных свойств на концентрацию хлороформа, данные фиксировались ежедневно в течение трех лет на одной из городских станций водоподготовки. Параметры, выбранные в качестве входных переменных, независимы друг от друга, максимальный коэффициент корреляции между ними не превышает 0,25.

Для решения поставленной задачи применяли пакет Neural Networks Toolbox программной среды MATLAB. Выбор структуры НС определяли в соответствии со сложностью и особенностями задачи.

При создании нейросети необходимо принимать во внимание, что общее число связей сети должно быть в разы меньше объема обучающей выборки, чтобы обеспечить достаточно гладкую аппроксимацию данных. Иначе нейросетевая модель просто "переобучится", то есть запомнит данные, но не сумеет сделать статистически значимый прогноз по новым значениям [8]. В нашей задаче для моделирования концентраций хлороформа имеется 50 связей, а объем выборки равен 6556, то есть сформулированное выше условие удовлетворяется.

Для определения концентраций хлороформа и создания условий максимально безопасного водопотребления проводился выбор типа искусственных нейронных сетей. Наилучшим оказался тип feed-forward backprop с прямой передачей сигнала и с обратным распространением ошибки. На рис. 1 приведены фрагменты выходного файла MATLAB, характеризующие параметры сети.

При создании сети подбирали оптимальные: функции обучения и число нейронов. Оптимальной оказалась функция LEARNPGDM, реализующая процесс обучения, а в качестве функции активации использовали дифференцируемую функцию TANSIG. Для оптимизации применили метод Левенберга-Марквардта [9].

На рис. 1а показан графический вывод корреляции в виде точечной диаграммы с набором таких целевых данных, как 

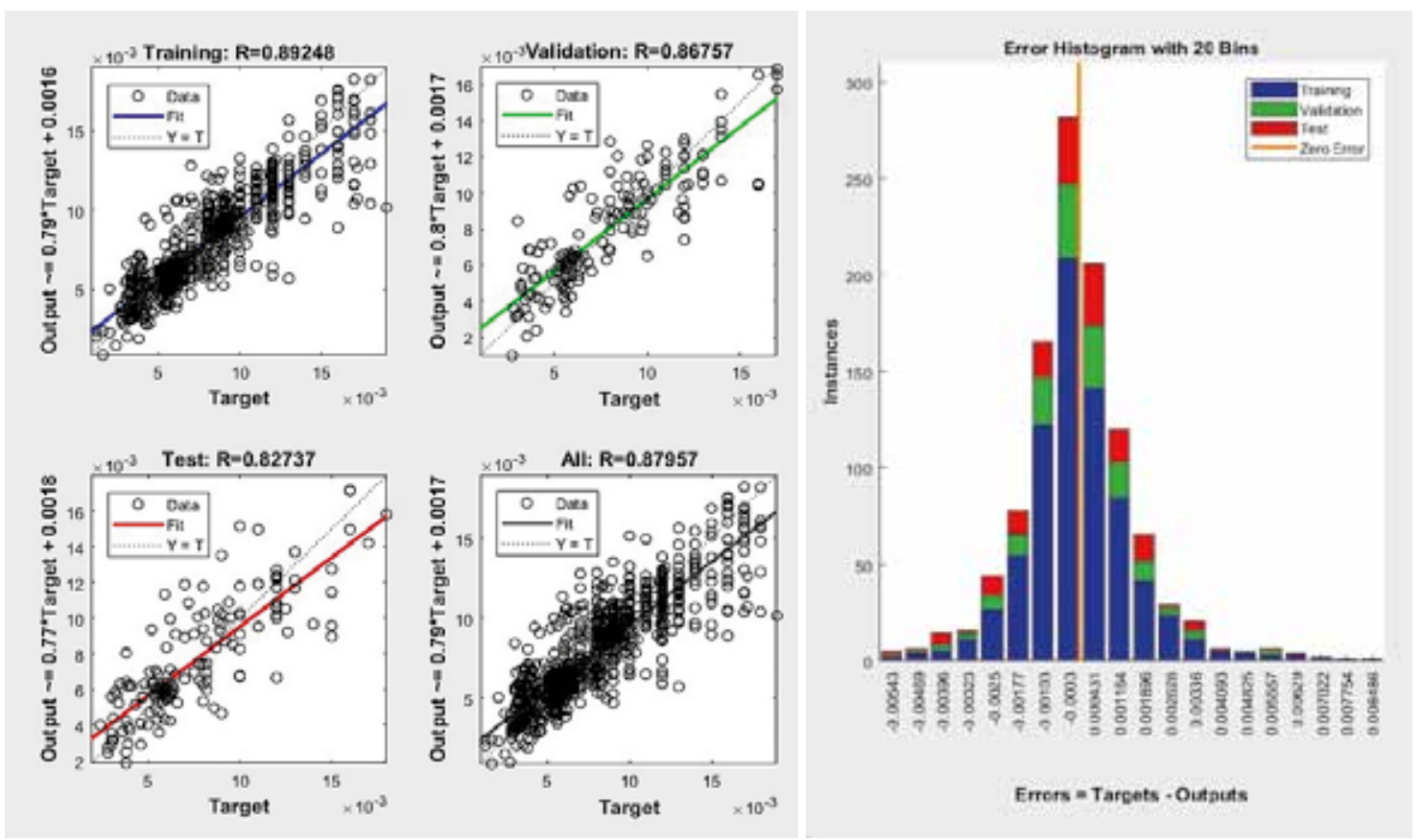

Puc. 1. Фрагменты выходного файла MATLAB, характеризующие параметры сети

"Цели" (Targets), "Выходы» (Outputs), "Ошибки" (Errors) для следующих выборок входных данных: обучающей (Training), контрольной (Validation), тестовой (Test) и для всего массива данных (All). На рис. 16 отображена гистограмма ошибок для проанализированных выборок.

Построенная сеть способна анализировать данные и формировать связи между показателями питьевой воды (входные параметры модели) и концентрацией хлорорганических соединений, образуемых в процессе обеззараживания, на примере хлороформа (выход модели).

Хлороформ выбран в качестве выходного параметра, так как по результатам проведенной оценки именно его концентрация вносит основной вклад в общее содержание хлорорганических соединений в питьевой воде на выбранном объекте исследования [10-11].

В качестве входных параметров выбраны следующие:

- температура воды, ${ }^{\circ} \mathrm{C}$;

- водородный показатель, ед. $\mathrm{pH}$;

- перманганатная окисляемость, мг/дм;

- хлоропоглощаемость, мг/дм³;

- остаточныйхлор, мг/дм³.

Выходной параметр - концентрация хлороформа, образованного в ходе обеззараживания питьевой воды. Учитывая показатели исходной воды, составим более подробную схему, описывающую работу нейронной сети (рис. 2).

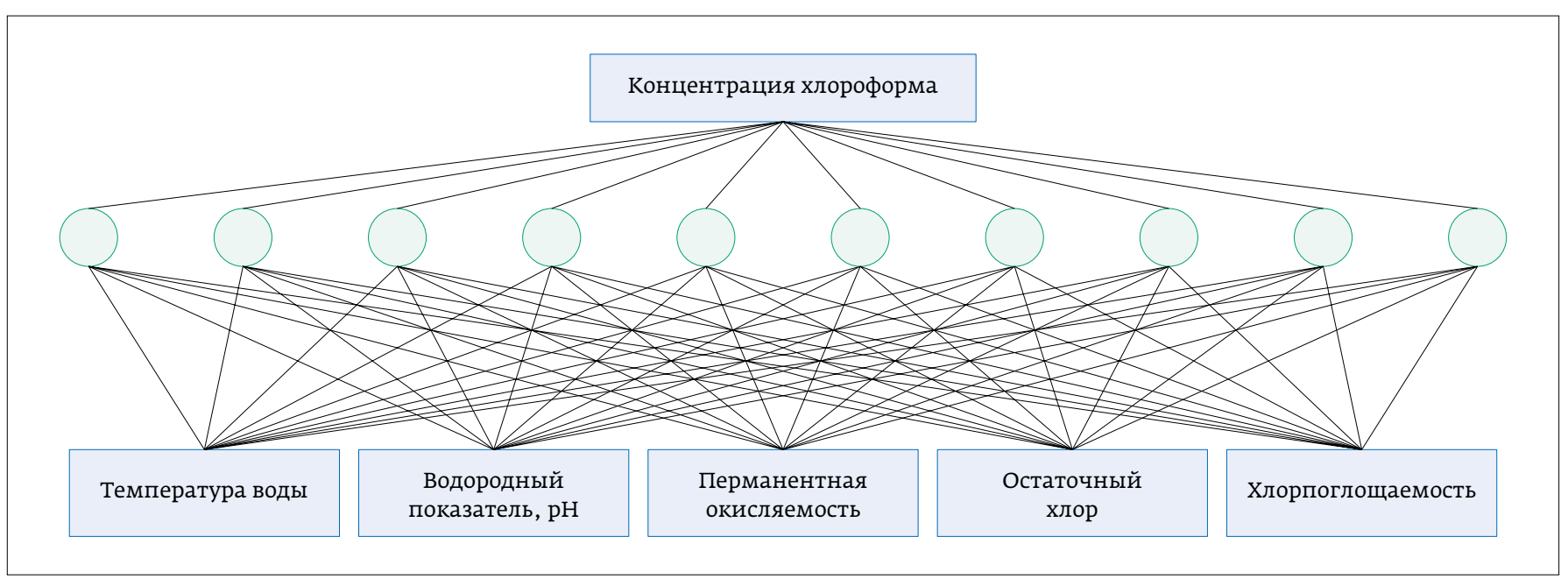

Puc. 2. Структура нейронной сети для концентрации хлороформа с учетом входных параметров 


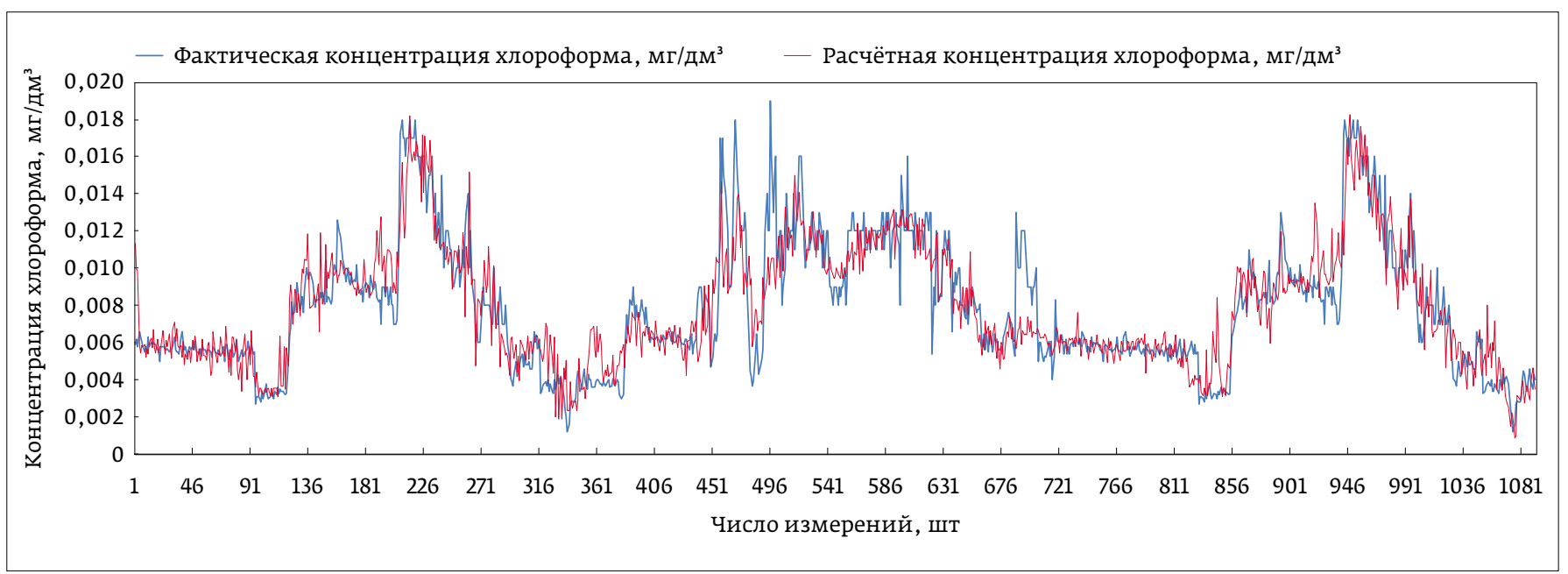

Puc. 3. Сравнение результатов моделирования и фактических значений для целевого параметра сети

\section{РЕЗУЛЬТАТЫ МОДЕЛИРОВАНИЯ И ОБСУЖДЕНИЕ}

По результатам моделирования построен график сравнения фактических, полученных методом газожидкостной хроматографии, и расчетных значений для выходного параметра сети - концентрации хлороформа (рис. 3).

Для анализа степени статистической зависимости и оценки достоверности полученных результатов определен коэффициент корреляции между расчетными и фактическими значениями. Сеть, показавшая наилучший результат обучения, имеет коэффициент корреляции 0,88.

Для исследования влияния отдельных факторов на целевой параметр проведена серия компьютерных экспериментов, диапазоны изменения параметров приведены в табл. 1.

Результаты экспериментов показаны на рис. 4(а-д). Выбрано два уровня фиксированных значений параметров: средний (синий график) - со значением концентрации хлороформа 0,0055 мг/дм³ и повышенный (красный график) $0,016 \mathrm{мг} /$ дм$^{3}$.

Во всех случаях зависимости концентрации хлороформа от факторов оказались полиномиальными с наличием минимумов и максимумом в исследуемых диапазонах. Характер зависимости концентрации хлороформа от температуры сохранялся в обоих случаях. При среднем уровне для окисляемости, хлорпоглощаемости и остаточного хлора обнаружены минимумы, наибольший разброс концентраций хлороформа наблюдался для остаточного хлора и составил 0,012 мг/ дм³. При увеличении значений $\mathrm{pH}$ идет снижение концентрации хлороформа.

При повышенном уровне рН изменение концентрации хлороформа в зависимости от окисляемости, хлорпоглощаемости и остаточного хлора было небольшим и составило не более 0,003 мг / дм³. При значениях рН свыше восьми единиц значение целевого параметра практически не изменяется.

\section{ЗАКЛЮЧЕНИЕ}

Для определения концентраций галогенорганических соединений на практике используются газохроматографические методы [12], которые не подходят для экспресс-анализа. Поэтому актуальной остается проблема прогнозирования содержания хлорорганических соединений в воде. Решение этого вопроса, по нашему мнению, лежит в области разработки нейронных сетей. В ходе исследования предложена нейросетевая модель, прогнозирующая концентрации хлороформа, который образуется во время обеззараживания питьевой воды, в зависимости от температуры, водородного показателя, перманганатной окислямости, хлорпоглощаемости и остаточного хлора. Коэффициент корреляции между фактическими и рассчитанными по модели значениями целевого показателя составил 0,88. С помощью построенной модели можно в режиме реального времени отслеживать концентрации хлорорганических

Таблица 1. Диапазоны изменения входных параметров

\begin{tabular}{|c|c|c|c|c|c|}
\hline & Температура, ${ }^{\circ} \mathrm{C}$ & $\mathrm{pH}$ & $\begin{array}{c}\text { Перманганатная } \\
\text { окисляемость, } \\
\text { мг/ } \text { дм }^{3}\end{array}$ & $\begin{array}{c}\text { Хлорпоглощаемость, } \\
\text { мг/ } \text { дм }^{3}\end{array}$ & $\begin{array}{c}\text { Среднесуточный } \\
\text { остаточный хлор, } \\
\text { мг/ } \text { дм }^{3}\end{array}$ \\
\hline $\min$ & 1 & 6,0 & 1,50 & 0,90 & 0,80 \\
\hline $\max$ & 30 & 8,9 & 8,75 & 2,64 & 2,54 \\
\hline
\end{tabular}




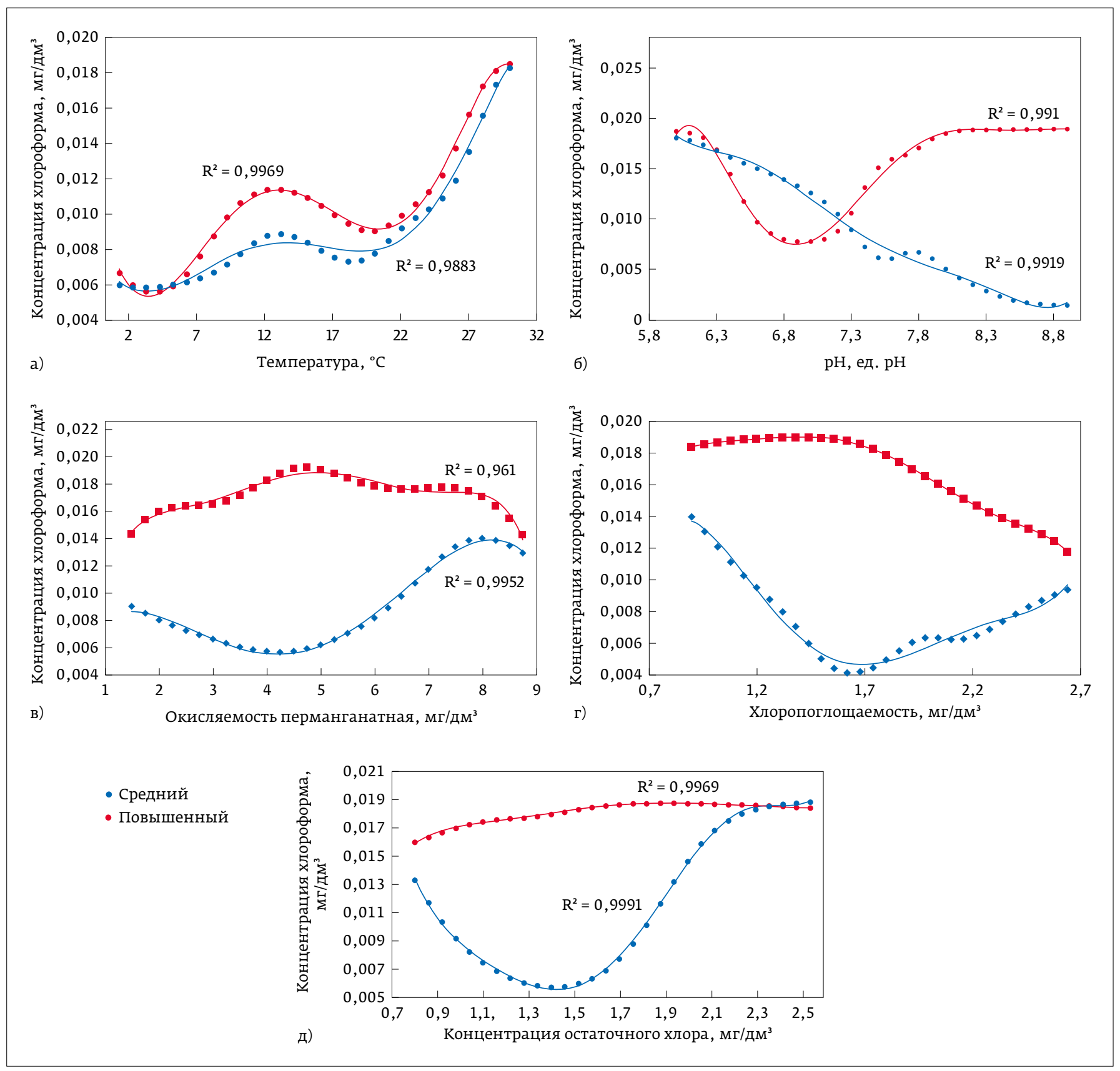

Puс. 4. зависимость концентрации хпороформа от показателей качества воды: а - температуры; 6 -водородного показателя рН; в - перманганатной окисляемости; г - хиорпоглощаемости; д-остаточного хлора

соединений, что позволит автоматизировать систему контроля процесса обеззараживания и снизить уровень канцерогенного риска употребления населением питьевой воды.

Исследование выполнено при финансовой поддержке РФФИ в рамках научного проекта № 18-35-00489.

\section{ЛИТЕРАТУРА}

1. Холова А.Р., Вагнер Е.В.,. Вождаева М.Ю., Кантор Е. А. и др. Современный подход к оценке качества питьевой воды // Экологическая, промышленная и энергетическая безопасность, 2017. С. 1463-1467.
2. Интегральная оценка питьевой воды централизованных систем водоснабжения по показателям химической безвредности: Методические рекомендации. М.: Федеральный центр гигиены и эпидемиологии Роспотребнадзора, 2012, 31 c.

3. Chen X., Feng F., Wu J., \& Liu W. Anomaly detection for drinking water quality via deep bilSTM ensemble // Paper presented at the GECCO 2018 Companion - Proceedings of the 2018 Genetic and Evolutionary Computation Conference Companion. 2018. 3-4. doi:10.1145/3205651.3208203.

4. Ahn J.C., Kim Y.W., Lee K.S., \& Koo J.Y. Residual chlorine management in water distribution systems using network modelling techniques: Case study in Seoul city // Water Science and Technology: Water Supply. 2004. 4(5-6). P. 421-429. 
5. Delpla I., Florea M., Rodriguez M.J.Drinking Water Source Monitoring Using Early Warning Systems Based on Data Mining Techniques // Water Resources Management. 2018. 33 (1). Р. 129-140.

6. Пономарев д.С. Нейрорегрессионная модель дезодорации воды на основе многослойного персептрона // Вестник КГТУ имени А. Н. Туполева. 2015. № 4. С. 16-19.

7. Червяков Н. И., Тихонов Э.Е. Применение нейронных сетей для задач прогнозирования и проблемы идентификации моделей прогнозирования на нейронных сетях // Нейрокомпьютеры: разработка, применение. 2003. № 10-11. С. 25-31.

8. Головко В.А. Нейронные сети: обучение, организация и применение Учебное пособие для вузов. - М.: ИПРЖР, 2001. 256 с

9. Усков А.А., Кузьмин А. В. Интеллектуальные технологии управления Искусственные нейронные сети и нечеткая логика. М.: Горячая линия Телеком, 2004. 143 с

10. Бахмацкая А. И., Плуготаренко Н. К. Анализ экологической безопас ности процессов хлорирования воды // Экология промышленного производства. 2016. № 2 (94). С. 52-56.

11. Юхно А.И., Плуготаренко Н.К. Анализ образования хлорпроизводных соединений в питьевой воде // Научный интернет-журнал "Технологии техносферной безопасности". 2018. № 3 (79). С. 28-35.

12. Кириченко В.Е., Первова М.Г., Пашкевич. К.И. Галогенорганические соединения в питьевой воде и методы их определения // Российский химический журнал. 2002. T. XLVI. № 4. С. 18-27.

\section{REFERENCES}

1. Holova A.R., Vagner E.V., Vozhdaeva M.Ju., Kantor E. A. and etc. Modern Approach to Assessing the Quality of Drinking Water // Jekologicheskaja promyshlennaja ijenergeticheskaja bezopasnost - Environmental, Industrial and Energy Security. 2017. PP. 1463-1467.

2. Integral Assessment of Drinking Water of Centralized Water Supply Systems by Chemical Safety Indicators: Guidelines. Moscow, Federal Center for Hygiene and Epidemiology of Rospotrebnadzor Publ. 2012
3. Chen X., Feng F., Wu J., \& Liu W. . Anomaly Detection for Drinking Water Quality via Deep bilSTM Ensemble // Paper presented at the GECCO 2018 Companion. Proceedings of the 2018 Genetic and Evolutionary Computation Conference Companion. 2018. PP. 3-4.

4. Ahn J.C., Kim Y.W., Lee K.S., \& Koo J.Y. Residual Chlorine Management in Water Distribution Systems Using Network Modelling Techniques: Case Study in Seoul City // Water Science and Technology: Water Supply. 2004 No. 4(5-6). PP. 421-429.

5. Delpla, I., Florea, M., Rodriguez, M.J. Drinking Water Source Monitoring Using Early Warning Systems Based on Data Mining Techniques // Water Resources Management. 2018. No. 33 (1). PP. 129-140.

6. Ponomarev D.S. Neuroregressive Model of Water Deodorization Based on Multilayer Perceptron // Vestnik KGTU imeni A. N. Tupoleva-Journal KNRTU after A. N. Tupolev. 2015. No. 4. PP. 16-19.

7. Chervjakov N.I., Tihonov Je. E. Application of Neural Networks for Forecasting Problems and Problems of Identification of Forecasting Models on Neural Networks // Nejrokomp'jutery: razrabotka, primenenieNeurocomputers: development, application. 2003. No. 10-11. PP. 25-31.

8. Golovko V.A. Neural Networks: Learning, Organization and Application Tutorial for Universities. Moscow, IPRZhR Publ. 2001.

9. Uskov A. A., Kuz'min A. V. Intelligent Control Technology. Artificial Neural Networks and Fuzzy Logic. Moscow, Hotline - Telecom Publ. 2004

10. Bachmackaja A.I., Plugotarenko N.K. The Study of Probability and the Control of Toxic Substances During the Chlorination of Water // Jekologija promyshlennogo proizvodstva-Industrial ecology. 2016. No. 2(94) PP. 52-56

11. Yuhno A. I., Plugotarenko N. K. Analysis of the Formation of Organochlorine Compounds in Drinking Water // Tehnologii tehnosfernoj bezopasnosti Technology of technosphere safety. 2018. No. 3 (79). PP. 28-35.

12. Kirichenko V.E., Pervova M.G., Pashkevich. K.I. Organohalogen Compounds in Drinking Water and Methods for their Determination // Rossijskij himicheskij zhurnal-Russian Chemical Journal. 2002. XLVI. № 4 PP. 18-27.

\section{РАМАНОВСКАЯ СПЕКТРОСКОПИЯ С АТОМНЫМ РАЗРЕШЕНИЕМ}

Рамановская спектроскопия широко используется на производстве для контроля качества и в научных исследованиях для изучения молекулярных процессов. В этом методе детектируется рассеянный свет, образующийся при взаимодействии молекул образца с излучением определенной частоты, например, от лазера. Однако нормальные колебательные моды приходится рассчитывать теоретически, т. к. их визуализация невозможна. Для этого требуется пространственное разрешение в несколько ангстрем, что почти на три порядка меньше дифракционного предела в оптике. Около 40 лет назад исследователи обнаружили, что

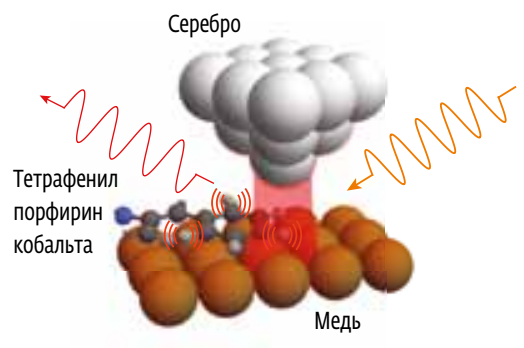

рамановский сигнал можно усилить в миллион раз, если использовать в качестве подложки металлическую поверхность (из серебра или золота). Лазер возбуждает атомы металла, усиливая сигнал от исследуемых молекул. Следующим шагом стало использование микроскопической металлической иглы, около которой фиксируется интересующая молекула. В такой модификации метода ученые записывают спектры с разрешением в 20-30 нм. Американские ученые преодолели это ограничение и получили уникальные спектры различных фрагментов одной молекулы с разрешением в несколько ангстрем.

Исследователи нанесли раствор тетрафенил порфирин кобальта на медную пластину и поместили серебряную иглу сканирующего туннельного микроскопа на расстоянии нескольких ангстрем от цели. Чтобы достичь атомного разрешения, эксперимент проводили в среде с очень высоким вакуумом и температурой $-267^{\circ} \mathrm{C}$. В таких условиях молекула порфирина крепко "примерзает» к медной поверхности, и иглу микроскопа можно перемещать вдоль образца на расстоянии около 0,2 нм.

Исследователи зафиксировали различия в частотах и построили карту интенсивности рамановского излучения в различных частях молекулы. Впервые стало возможно наблюдать колебательные спектры молекулы, которые ранее рассчитывались исключительно теоретически.

Ученым предстоит разрешить несколько проблем на пути широкого использования разработанного метода. Экстремально низкие вакуум и температура доступны для воспроизведения в немногих лабораториях мира. Неизвестно, смогут ли комбинации вещество/металлическая подложка - иные, чем порфирин кобальта/ медь - обеспечить необходимое сильное взаимодействие, которое определило успех эксперимента. Также необходимо изучить, искажает ли металлическая поверхность колебательное состояние молекулы. Преодоление этих ограничений откроет уникальные возможности в исследовании и визуализации молекул.

Nature, 2019. DOI: 10.1038/s41586-019-1059-9 

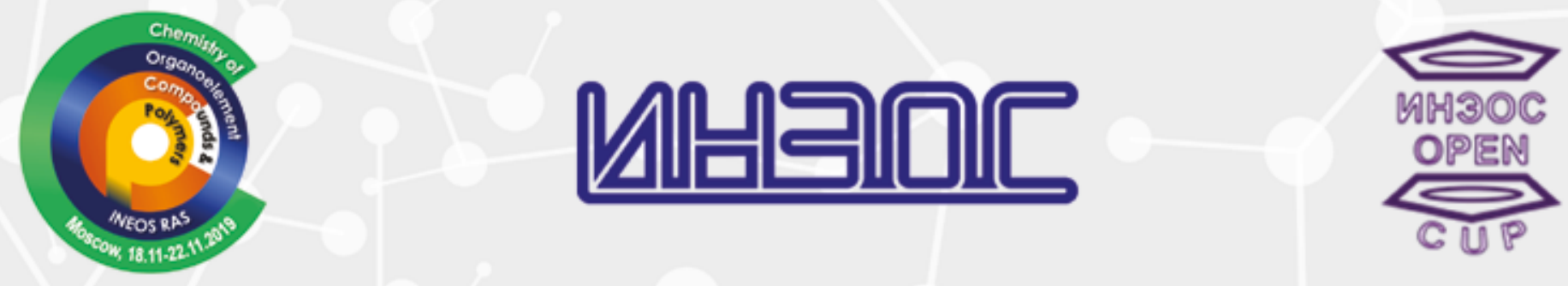

Институт элементоорганических соединений им. А.Н. Несмеянова РАН приглашает ученых и представителей бизнес-сообщества принять участие в

\section{Юбилейной межАунароАной конференции, ПОСвящеНной 65-^етию ИНЭОС РАН и 120-летию СО Аня рОЖАения वкаАемика А. Н. Несмеянова}

\section{8-22 ноября 2019 года в ИНЭОС РАН}

Целью конференции является обсуждение последних достижений и обмен опытом между ведущими российскими и зарубежными учеными в области химии элементоорганических соединений и полимерных материалов. С пленарными лекииями согласились выступить ведущие российские и зарубежные ученые: члены Российской академии наук А.Р. Хохлов, И.Л. Еременко, И.Л. Федюшкин, С.Н. Калмыков, профессора K. Matyjaszewski (США), S.P. Nolan (Бельгия), P. Chirik (США), C. Copéret (Швейцария), T. Yokozawa (Япония) и др. В рамках конферениии пройдет традиционный ежегодный открытый конкурс научных работ "ИНЭОС ОРЕN CUP 2019". Для участия в конкурсе принимаются экспериментальные и теоретические работы в области химии элементоорганических соединений, высокомолекулярных соединений, органической химии, а также физико-химических методов исследования строения вещества. Работы для участия в конкурсе "ИНЭОС ОРЕN СUР" проходят экспертизу независимым жюри конкурса, которое также отбирает работы для публикации в специальном сборнике "ИНЭОС ОРЕN SELECT". Работы, имеющие выраженные прик^адные перспективы, будут выАелены в отАеАьную сессию очной части конкурса «ИНЭОС ОРEN APPLIED», после которой 22 ноября 2018 гоАа состоится крУгАый стол «МетаАмокомп^ексный катаАиз: от теории к практике». ПобеАители конкурса буАут отмечены Аенежными премиями.

Подробная информация о конференции и о конкурсе - на сайте www.ineos.ac.ru

СПОНСОРЫ КОНФЕРЕНЦИИ:

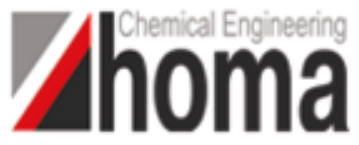

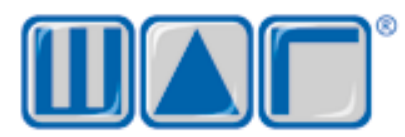

ИНФОРМАЦИОННЫЕ ПАРТНЕРЫ:

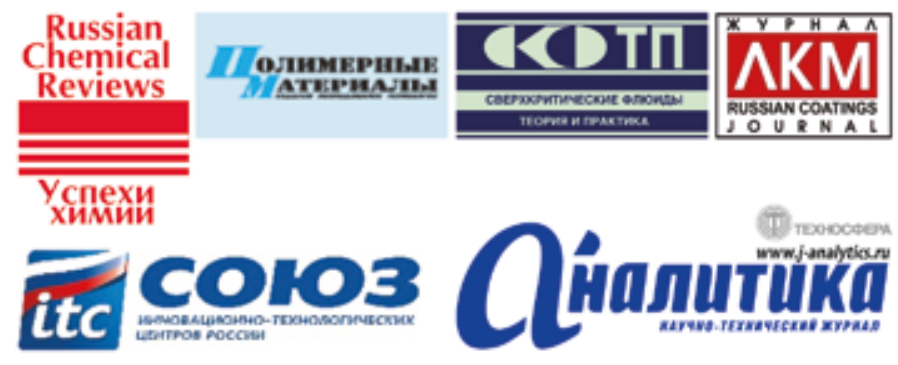

